\title{
The Characteristic of Nurses' Eye Movements during Observation of Patients with Disturbed Consciousness
}

\author{
Ryoko Tsuchiya', Mayumi Shimizu², Kasumi Mikami³, Keiko Aizuㄹ, Yuichi Hirakawa3, \\ Sonoko Takase ${ }^{4}$, Yoshiko Nishizawa ${ }^{3}$ \\ ${ }^{1}$ Hirosaki University Hospital, Hirosaki University, Hirosaki, Japan \\ ${ }^{2}$ Institute of Radiation Emergency Medicine, Hirosaki University, Hirosaki, Japan \\ ${ }^{3}$ Graduate School of Health Sciences, Hirosaki University, Hirosaki, Japan \\ ${ }^{4}$ Doctoral Program, Graduate School of Health Sciences, Hirosaki University, Hirosaki, Japan \\ Email: ryoko32350@hirosaki-u.ac.jp
}

How to cite this paper: Tsuchiya, R., Shimizu, M., Mikami, K., Aizu, K., Hirakawa, Y., Takase, S. and Nishizawa, Y. (2017) The Characteristic of Nurses' Eye Movements during Observation of Patients with Disturbed Consciousness. Open Journal of Nursing, 7, 1502-1514.

https://doi.org/10.4236/ojn.2017.712105

Received: November 28, 2017 Accepted: December 25, 2017

Published: December 28, 2017

Copyright () 2017 by authors and Scientific Research Publishing Inc. This work is licensed under the Creative Commons Attribution International License (CC BY 4.0).

http://creativecommons.org/licenses/by/4.0/

\begin{abstract}
Objective: The objective of this study was to clarify the characteristics of nurses' eye movements during observation of patients with disturbed consciousness by comparing intuition ability, critical thinking, and clinical experience years. Methods: Participants were 19 nurses working on a neurosurgery ward in Japan and caring for patients with consciousness disturbance who were unable to speak. Ten novice nurses $(24.8 \pm 3.36$ years old $)$ and nine expert nurses ( $38.3 \pm 5.77$ years old) were compared. The observation scene, displayed on a computer screen, was a static image of a simulated patient in a resting state who had developed right putaminal hemorrhage. We showed the participants an information sheet about the simulated patient then used an eye tracking camera to analyze gaze points, gaze time, and gaze count during observation. The Kuroda Intrinsic Intuitive Ability Scale (KIIS) created by Kawahara and the Critical Thinking Disposition Scale created by Hirayama were used for measuring intrinsic intuition ability and critical thinking disposition. Results: There were no significant differences between observation time of novices $(5.0 \pm 2.01$ minutes) and experts ( $5.4 \pm 1.78$ minutes), nor between their total gaze time and total count. The proportion of gazing at the monitor by novice nurses and gazing at the face and bed fences by expert nurses tended to be high. The KIIS general education score influenced the gaze time and gaze count during simulated patient observation. The KIIS logical thinking score affected the gaze count to the simulated patient's face. Conclusion: There was no significant difference between the total gaze time and the total count of novices and experts. The KIIS score affected the gaze during observation of the simulated patient.
\end{abstract}




\section{Keywords}

Nursing Observation, Eye Movement, Critical Thinking,

Patient with Disturbed Consciousness

\section{Introduction}

Nurses decide the patient's problem and the priorities of care based on patients' complaints and information obtained by observation when they care for patients. Nurses need to have insight during evaluation of patients with disturbed consciousness because they cannot complain about their pain and symptoms. Until now, we have been investigating the actual situation of nurses' observation during evaluation of patients with disturbed consciousness. In a previous study, we analyzed nursing records because nursing records show clinical judgment and the contents of nursing practice. In the results of the study, the nursing records mainly described the result of checking the monitor. It was difficult for nurses to observe the facial expression of patients with disturbed consciousness, so most of the patients' facial expressions were not described in the nursing records [1]. However, we suspected that nurses may not have recorded all that they observed. Then, we investigated by interview, in order to clarify the actual condition of nurses' observation. In that study, nurses observed patients with disturbed consciousness while thinking of patients' changes. However, nurses experienced difficulty because they did not have any confidence in observing and judging patients' changes [2]. From our studies, the outline of the nurses' observation during evaluation of patients with disturbed consciousness is clear. However, we have not investigated the process and points of view.

To watch something for more than a set time with the fovea centralisis called "gaze." The method of measuring eye movements is used as a method for estimating the viewing target [3]. Eye tracking technology in nursing research is used to measure nurses' eye movements, such as for evaluation in simulation education, eye movements for surgical assistance, and patient observation [4] [5] [6] [7] [8]. Therefore, it was thought that we could clarify the nurses' point of view and the process of observation during evaluation of patients with disturbed consciousness by measuring their eye movement and gaze point.

When expert nurses observe patients, they may notice something unusual about the patient without clear grounds, objective data, or patient's complaints. Such a nurse's sense without a scientific basis is called "intuition." Especially, Benner said intuition was a characteristic ability only of experienced expert nurses [9]. Novice nurses do not have enough necessary experience to judge whether the patient's symptoms need attention, if closer care is required in the future, or it is considered to be no problem. On the other hand, expert nurses have enough experience. Therefore, Benner said that expert nurses can intuitively grasp the situation and focus on the exact problem area without inappro- 
priate nursing diagnosis and care [9]. Based on these facts, we thought that the nurses' intuition ability during evaluation of patients with disturbed consciousness influences the nurses' attention to points considered as problems, that is their gaze.

Ueno et al. investigated the characteristics of nursing practice skills, and reported that nurses with 3 to 5 years of clinical experience are at a competent level, but their nursing practical skills are low. For example, these skills were "skill to judge, respond to change", "skill to cooperate with patients, families, and medical teams," "attitudes to prevent incidents and medical accidents", and "skill to refine self" [10]. Nurses are regularly transferred within the department. When experienced nurses are transferred to a department where previous knowledge cannot be applied, they temporarily can only practice beginner level skills. Consequently, in this study, we defined novice nurses as having less than 3 years of clinical experience, and expert nurses a shaving worked for more than 5 years in a neurosurgery ward with nursing experience of over 10 years.

Tonai and Miyakoshi reported that inference was a characteristic element in the clinical judgment of nurses, and inference means proficiency [11]. Expert nurses have logical inference skills, and as a result, they can use knowledge while predicting the outcome [12] [13]. When nurses are inferring, they are required to objectively perceive things, and think from a multifaceted and multilateral perspective. We thought that nurses' critical thinking influences clinical judgment of the area considered a problem during evaluation of patients with disturbed consciousness. Therefore, we focused on the nurses' eye movements to analyze their observations. We investigated the characteristics of nurses' eye movements during observation of patients with disturbed consciousness by comparing novice nurses with expert nurses.

\section{Purpose}

The objective of this study was to clarify the characteristics of nurses' eye movements during observation of patients with disturbed consciousness by comparing intuition ability, critical thinking, and clinical experience years.

\section{Method}

\subsection{Participants}

The participants in this study were novice and expert nurses working on a neurosurgery ward and caring for patients with consciousness disturbance who were unable to speak in two hospitals of Japan. We excluded participants who wear glasses because the eye-tracker measures eye movements by the pupil/corneal reflection system, and there is a possibility that the viewpoint cannot be displayed by the refractive index of the lens when a nurse wears glasses.

We explained the purpose and method of this study to the nurse departments and managers of the two hospitals and asked for selection of participants. We conducted our study after receiving informed consent from all participants. 


\subsection{Observation Scene}

The observation scene was a static image of the rest scene of a simulated patient (Figure 1). The simulated patient was a right putaminal hemorrhage patient diagnosed with hypertension but who had stopped taking antihypertensive medicine by self-judgment. The scene was the 5th day after having a right put aminal hemorrhage. The simulated patient lay down on the bed, an electrocardiogram monitor, an automatic sphygmomanometer, and a pulse oximeter were attached to the right upper limb, dripping infusion from the central venous catheter of the right subclavian vein, and inserting a bladder indwelling catheter. In clinical situations, when a nurse wants to observe the patient carefully, the nurse observes while approaching the patient. Therefore, the static image was programmed so that the enlarged static image was displayed by moving the cursor on the part that they wanted to observe carefully when a created static image was displayed on a personal computer. In addition, for the participants to collect information on simulated patients, we created an information sheet describing the basic information of the simulated patient and the course of treatment, an instruction book, an injection instruction note, and a worksheet as memo paper when collecting information. We confirmed the validity of the setting of the case and the created static image with the Certified Nurse in Stroke Rehabilitation Nursing $(\mathrm{CN})$ and neurosurgical specialist in Japan.

\subsection{Methods of Data Collection}

\subsubsection{Measurement of Participants' Eye Movements}

The created static image was displayed on a touch panel type monitor, and we measured participants' eye movements when they observed the simulated patient. Eye movements were measured with an Eye Mark recorder EMR-9 manufactured by the NAC company, which is capable of acting as a visual field camera and a pupil camera. We performed calibration at nine locations: three each at the top, at the middle, and at the bottom.

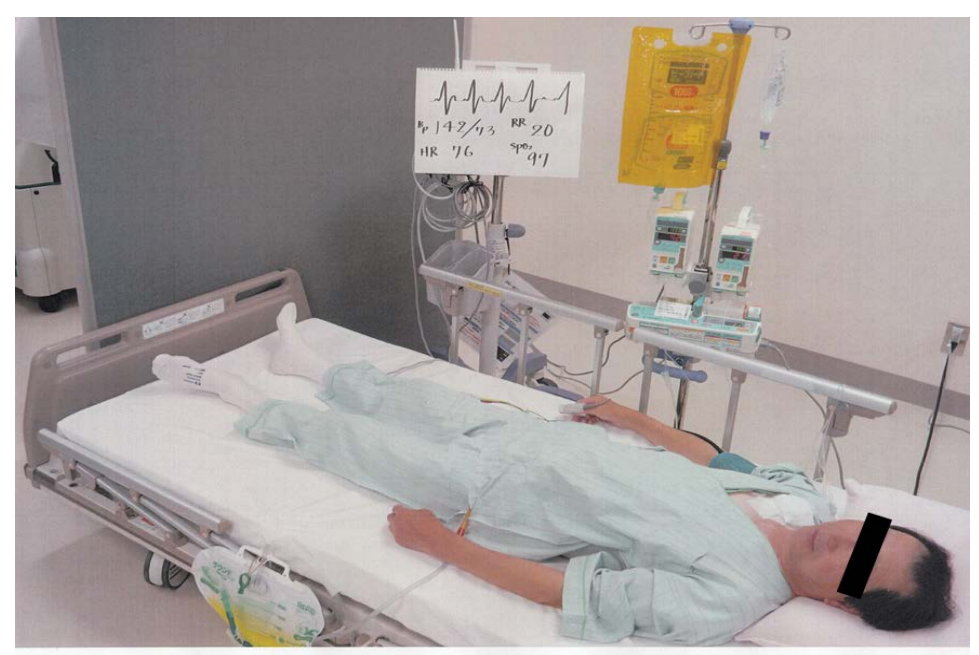

Figure 1. Observation scene. 


\subsubsection{The Instructions for Participants}

We showed participants the information sheet about the simulated patient and asked them to collect information about the simulated patient within $10 \mathrm{mi}$ nutes. After that, we told participants, "You are in charge of the simulated patient. As the first visit during the day, you visited this patient's room. Please observe what you need to care for the simulated patient within 8 minutes. When you want to observe the simulated patient carefully, touch the part you want to observe. Then the image will become bigger and once you touch it again, it will return to normal." We set the information collection time and observation time. To determine these times, we referred to previous studies and the time taken for visiting a patient in the first visits during the day of three day nurses working on a neurosurgery ward [14] [15].

\subsubsection{Measurement of Participants' Intrinsic Intuition Ability}

We used the Kuroda intrinsic intuitive ability scale (KIIS) created by Kawahara to measure the intuition ability of clinical nurses [16]. The scale consists of 27 items, and 6 subscales for intelligence, expertise, logical thinking, involvement, sensitivity, and general education. Each item was ranked at five stages from 1 (strongly disagree) to 5 (strongly agree). The scale score was $27-135$ points. The Cronbach's $\alpha$ factor of the scale was 0.90 .

\subsubsection{Measurement of Participants' Critical Thinking Disposition}

We used the critical thinking disposition scale created by Hirayama and Kusumi to measure the critical thinking disposition of clinical nurses [17]. The scale was created by integrating a scale for measuring thought trends and attitudes. The scale consisted of 33 items, and 4 subscales of consciousness to logical thinking, spirit of inquiry, objectivity, and emphasis on evidence. Each item was ranked at five stages from 1 (strongly disagree) to 5 (strongly agree). The scale score was 33 - 165 points. The Cronbach's $\alpha$ factor of the 4 subscales was $0.57-0.85$.

\subsection{Study Period}

This study was conducted from August 2016 to July 2017.

\subsection{Method of Data Analysis}

To analyze participants' eye movements, the exclusive eye mark data analysis software EMR-d Factory Ver 2.1 manufactured by NAC Inc. was used. We defined gaze items while consulting with the $\mathrm{CN}$. The items were patient's face, left and right upper and lower limbs, chest and abdomen, drip route, monitor, urine bag, insertion and connection part of bladder indwelling catheter, part of the elastic stocking, part of blood pressure, pulse oximeter, worksheet and paper of injection instruction, and bed fence. We displayed the eye mark on the static image and calculated the gaze time, the total gaze time, the gaze count, and the total gaze count. The gaze time means the time gazing at a gaze item, the total gaze time means the sum of the gaze times, the gaze count means the number of times gazing at a gaze item, and the total gaze count means the sum of the gaze 
counts. In addition, we defined the time not gazing as the difference between the total gaze time and the observation time. Based on the report by Daikoku and Saito, we defined a gaze as when the eye mark stayed for more than 0.1 seconds [8].

\section{Statistical Method}

The differences in gaze duration between novice nurses and expert nurses was compared with unpaired t-tests and multiple logistic regression analysis. The factors influencing gaze duration were analyzed by stepwise multiple regression analysis. For statistical analysis, we used SPSS Statistics 21.0, and the statistical significance level was set at 0.05 .

\subsection{Ethical Considerations}

This study was approved be the Ethics Committee of Hirosaki University Graduate School of Health Science, Hirosaki, Japan (approval number: 2015-020). Candidate participants were given explanations about the study purpose and methods, details that would be requested, protection of privacy, and freedom to discontinue the study. Verbal and written consent were also obtained from participants before research.

\section{Results}

\subsection{Summary of Participants}

The participants were 10 novice and 9 expert nurses. The average age of novice nurses was $24.8 \pm 3.36$ years, the average length of clinical experience was $1.5 \pm$ 0.56 years, and the length of experience on a neurosurgery ward was $1.5 \pm 0.26$ years. On the other hand, the average age of expert nurses was $38.3 \pm 5.77$ years, the average length of clinical experience was $15.5 \pm 3.21$ years, and the length of experience on a neurosurgery ward was $10.3 \pm 2.4$ years.

In the educational background of the novice nurses, six nurses had completed nursing college, three a nursing vocational school 3-year course, and two a 5 -year consistent course in high school. On the other hand, the educational background of the expert nurses was that two nurses had completed nursing junior college, five a nursing vocational school 3-year course, and one a nursing vocational school 2-year course.

\subsection{Time of Information Collection and Observation}

The average time taken to collect information on the simulated patient by the novice nurses was $7.6 \pm 2.51$ minutes, and for the expert nurses was $7.3 \pm 2.08$ minutes; the average time observing the simulated patient by the novice nurses was $5.0 \pm 2.01$ minutes, and for the expert nurses was $5.4 \pm 1.78$ minutes. There was no significant difference between the novice nurses and the expert nurses during the time spent collecting information and observing the simulated patient. 


\subsection{The Intrinsic Intuition Ability and Critical Thinking Disposition of Participants}

Table 1 shows the KIIS scores of participants. The novice nurses' KIIS general education score was $7.3 \pm 2.00$ and the expert nurses' score was $9.9 \pm 3.10$. The novice nurses' general education score was significantly higher $(p<0.05)$. The expert nurses' total KIIS score and the expert nurses' scores of intelligence, logical thinking, expertise, involvement, and sensitivity were higher. However, there was no significant difference in these scores between the expert and novice nurses.

Table 2 shows the Critical Thinking Disposition Scale scores of participants. Total score of Critical thinking disposition scale, and scores of subcategories such as consciousness to logical thinking, emphasison evidence were higher in expert than novice nurses. Scores of subcategories of sprit of inquiry, objectivity were higher in novice than expertnurses. There was no significant difference between the novice and expert nurses in the Critical Thinking Disposition Scale score.

\subsection{Gaze Time}

Table 3 shows the gaze time of novice nurses and expert nurses. For the novice nurses, the total gaze time was $182.7 \pm 96.68$ seconds, and the time not gazing of gaze items was $116.7 \pm 96.68$ seconds. For the expert nurses, the total gaze time was $183.0 \pm 88.91$ seconds, and the time not gazing at gaze items was $142.2 \pm$ 62.99 seconds. There was no significant difference between the novice and expert nurses in the total gazing time, or the time not gazing items. When comparing gaze time of each gaze item between the novice and expert nurses, the gaze time of monitor was significantly longer by novice nurses $(p<0.05)$. However, there was no significant difference in the gaze time of other gaze items. There was no significant difference in the proportion of observation gaze time for the novice and expert nurses.

Table 1. Score of Kuroda intrinsic intuition ability scale $(M \pm S D)$.

\begin{tabular}{|c|c|c|c|c|c|c|c|c|}
\hline & & Intelligence & Expertise & $\begin{array}{l}\text { Logical } \\
\text { thinking }\end{array}$ & Involvement & Sensitivity & $\begin{array}{c}\text { General } \\
\text { education }\end{array}$ & total \\
\hline Novice nurses & $\mathrm{n}=10$ & $15.8 \pm 2.70$ & $14.0 \pm 2.31$ & $10.8 \pm 2.74$ & $14.0 \pm 2.05$ & $15.4 \pm 1.65$ & $7.3 \pm 2.00$ & $77.3 \pm 7.65$ \\
\hline Expert nurses & $\mathrm{n}=9$ & $18.8 \pm 4.06$ & $16.6 \pm 3.40$ & $11.3 \pm 2.96$ & $13.3 \pm 2.12$ & $14.8 \pm 1.72$ & $9.9 \pm 3.10$ & $84.7 \pm 9.90$ \\
\hline
\end{tabular}

Unpaired t-test. ${ }^{\star}: p<0.05$.

Table 2. Score of critical thinking disposition scale ( $M \pm S D)$.

\begin{tabular}{|c|c|c|c|c|c|c|}
\hline & & $\begin{array}{l}\text { Consciousness } \\
\text { to logical thinking }\end{array}$ & $\begin{array}{l}\text { Spirit of } \\
\text { inquiry }\end{array}$ & Objectivity & $\begin{array}{c}\text { Emphasis } \\
\text { on evidence }\end{array}$ & total \\
\hline Novice nurses & $\mathrm{n}=10$ & $33.7 \pm 5.7$ & $36.7 \pm 3.53$ & $25.9 \pm 1.97$ & $9.8 \pm 1.29$ & $106.1 \pm 6.89$ \\
\hline Expert nurses & $\mathrm{n}=9$ & $38.3 \pm 10.46$ & $35.8 \pm 5.33$ & $25.4 \pm 4.33$ & $10.4 \pm 1.94$ & $110.0 \pm 20.78$ \\
\hline
\end{tabular}


Table 3. Gaze time ( $\mathrm{M} \pm \mathrm{SD}$, seconds).

\begin{tabular}{|c|c|c|c|c|c|}
\hline Items of gaze & Novice nurses $(\mathrm{n}=10)$ & Expert nurses $(\mathrm{n}=9)$ & Items of gaze & Novice nurses $(\mathrm{n}=10)$ & Expert nurses $(n=9)$ \\
\hline \multirow[b]{2}{*}{ Face } & $24.2 \pm 26.56$ & $34.3 \pm 43.03$ & \multirow[b]{2}{*}{ Monitor } & $26.2 \pm 14.36$ & $14.3 \pm 6.41^{*}$ \\
\hline & $(7.7 \pm 7.51)$ & $(9.2 \pm 9.66)$ & & $(9.0 \pm 4.25)$ & $(5.1 \pm 4.63)$ \\
\hline \multirow{2}{*}{ Right upper limb } & $16.4 \pm 13.79$ & $12.3 \pm 6.39$ & \multirow{2}{*}{ Urine bag } & $7.3 \pm 5.15$ & $5.7 \pm 6.06$ \\
\hline & $(5.1 \pm 2.64)$ & $(4.1 \pm 2.65)$ & & $(2.7 \pm 2.23)$ & $(1.7 \pm 1.67)$ \\
\hline \multirow{2}{*}{ Right lower limb } & $8.4 \pm 3.73$ & $12.1 \pm 6.94$ & $\begin{array}{c}\text { Insertion and } \\
\text { connection part of }\end{array}$ & $1.4 \pm 3.39$ & $1.5 \pm 2.59$ \\
\hline & $(2.9 \pm 1.00)$ & $(4.0 \pm 2.19)$ & $\begin{array}{c}\text { bladder } \\
\text { indwelling catheter }\end{array}$ & $(0.7 \pm 1.91)$ & $(0.4 \pm 0.75)$ \\
\hline \multirow{2}{*}{ Left upper limb } & $10.2 \pm 8.60$ & $9.8 \pm 10.57$ & \multirow{2}{*}{$\begin{array}{l}\text { Part of the elastic } \\
\text { stocking }\end{array}$} & $0.4 \pm 1.35$ & $1.0 \pm 2.29$ \\
\hline & $(3.7 \pm 3.22)$ & $(2.9 \pm 2.25)$ & & $(0.2 \pm 0.64)$ & $(0.3 \pm 0.71)$ \\
\hline \multirow{2}{*}{ Left lower limb } & $11.2 \pm 7.63$ & $9.0 \pm 6.39$ & Part of blood & $0.0 \pm 0.08$ & $0.3 \pm 0.6$ \\
\hline & $(3.9 \pm 3.12)$ & $(2.9 \pm 1.82)$ & $\begin{array}{c}\text { pressure, } \\
\text { pulse oximeter }\end{array}$ & $(0.0 \pm 0.04)$ & $(0.1 \pm 0.20)$ \\
\hline \multirow{2}{*}{ Chest and abdomen } & $14.5 \pm 10.85$ & $12.4 \pm 8.26$ & \multirow{2}{*}{$\begin{array}{l}\text { Worksheet and } \\
\text { paper of injection } \\
\text { instruction }\end{array}$} & $18.4 \pm 40.68$ & $13.4 \pm 18.96$ \\
\hline & $(4.8 \pm 2.87)$ & $(3.5 \pm 2.22)$ & & $(5.6 \pm 10.89)$ & $(3.8 \pm 4.57)$ \\
\hline \multirow[b]{2}{*}{ Drip route } & $37.4 \pm 37.16$ & $44.5 \pm 15.86$ & \multirow[b]{2}{*}{ Bed fence } & $6.7 \pm 4.03$ & $12.4 \pm 12.51$ \\
\hline & $(11.1 \pm 7.2)$ & $(14.3 \pm 3.96)$ & & $(2.4 \pm 1.44)$ & $(4.2 \pm 4.16)$ \\
\hline Gaze time & $182.7 \pm 96.68$ & $183.0 \pm 88.91$ & $\begin{array}{l}\text { Time not gazing } \\
\text { of gaze items }\end{array}$ & $116.7 \pm 70.45$ & $142.2 \pm 62.99$ \\
\hline$(\%)$ & $(59.8 \pm 16.66)$ & $(56.3 \pm 15.22)$ & $(\%)$ & $(40.2 \pm 16.66)$ & $(43.7 \pm 15.22)$ \\
\hline
\end{tabular}

The percentage of the gaze time of each item at observation time: (\%). Comparison between novice nurses and expert nurses: multiple logistic regression analysis. ${ }^{*}: p<0.05$. 
Table 4. Gaze count $(M \pm S D$, times)

\begin{tabular}{|c|c|c|c|c|c|}
\hline Items of gaze & Novice nurses $(\mathrm{n}=10)$ & Expert nurses $(n=9)$ & Items of gaze & Novice nurses $(\mathrm{n}=10)$ & Expert nurses $(n=9)$ \\
\hline \multirow[b]{2}{*}{ Face } & $36.9 \pm 32.87$ & $50.4 \pm 57.14$ & \multirow[b]{2}{*}{ Monitor } & $31.3 \pm 17.66$ & $29.1 \pm 14.40$ \\
\hline & $(9.9 \pm 7.40)$ & $(11.1 \pm 10.23)$ & & $(9.4 \pm 5.04)$ & $(8.1 \pm 4.22)$ \\
\hline \multirow{2}{*}{ Right upper limb } & $40.7 \pm 24.61$ & $37.3 \pm 16.81$ & \multirow{2}{*}{ Urine bag } & $11.8 \pm 8.16$ & $10.6 \pm 11.41$ \\
\hline & $(11.8 \pm 5.28)$ & $(10.2 \pm 4.73)$ & & $(3.4 \pm 2.20)$ & $(2.5 \pm 2.29)$ \\
\hline \multirow{2}{*}{ Right lower limb } & $29.7 \pm 13.03$ & $33.9 \pm 17.82$ & \multirow{2}{*}{$\begin{array}{c}\text { Insertion and } \\
\text { connection part of } \\
\text { bladder indwelling } \\
\text { catheter }\end{array}$} & $1.9 \pm 2.69$ & $3.9 \pm 6.47$ \\
\hline & $(9.1 \pm 3.89)$ & $(8.8 \pm 3.42)$ & & $(0.6 \pm 1.09)$ & $(0.8 \pm 1.2)$ \\
\hline \multirow{2}{*}{ Left upper limb } & $25.0 \pm 17.58$ & $23.7 \pm 25.38$ & \multirow{2}{*}{$\begin{array}{l}\text { Part of the } \\
\text { elastic stocking }\end{array}$} & $0.7 \pm 2.21$ & $1.1 \pm 2.20$ \\
\hline & $(7.2 \pm 4.85)$ & $(5.7 \pm 3.98)$ & & $(0.2 \pm 0.74)$ & $(0.3 \pm 0.56)$ \\
\hline \multirow{2}{*}{ Left lower limb } & $31.1 \pm 20.72$ & $26.8 \pm 13.00$ & Part of blood & $0.2 \pm 0.42$ & $0.4 \pm 0.73$ \\
\hline & $(8.8 \pm 4.08)$ & $(7.3 \pm 3.59)$ & $\begin{array}{c}\text { pressure, } \\
\text { pulse oximeter }\end{array}$ & $(0.1 \pm 0.19)$ & $(0.1 \pm 0.22)$ \\
\hline \multirow{2}{*}{ Chest and abdomen } & $41.4 \pm 25.35$ & $39.8 \pm 25.54$ & \multirow{2}{*}{$\begin{array}{l}\text { Worksheet and } \\
\text { paper of injection } \\
\text { instruction }\end{array}$} & $15.3 \pm 31.89$ & $13.0 \pm 13.69$ \\
\hline & $(12.0 \pm 5.61)$ & $(9.3 \pm 4.52)$ & & $(3.9 \pm 6.87)$ & $(3.4 \pm 4.28)$ \\
\hline \multirow{2}{*}{ Drip route } & $59.5 \pm 35.09$ & $87.4 \pm 34.76$ & \multirow{2}{*}{ Bed fence } & $23.0 \pm 10.26$ & $36.1 \pm 23.71$ \\
\hline & $(16.6 \pm 7.41)$ & $(22.5 \pm 5.64)$ & & $(7.0 \pm 3.25)$ & $(9.9 \pm 6.80)$ \\
\hline
\end{tabular}

The percentage of the gaze count of each item at total gaze count: (\%). Comparison between novice nurses and expert nurses: multiple logistic regression analysis.

score of general education, which was a KIIS item, was selected as an independent variable $(p<0.05)$.

To analyze factors influencing total gaze count while observing the simulated patient, we used multiple regression analysis with total gaze count as a dependent variable by a similar method. The score of general education, which was a KIIS item, was selected as an independent variable $(p<0.05)$. There was a significant difference in the results of analysis of variance $(p<0.05)$, and $R^{2}=0.22$.

In our previous study, the patient's face was an item with less frequent observation by cliical nurses. To analyze factors influencing observation of the patient's face, we used stepwise multiple regression analysis with the gaze count of the patient's face as the dependent variable. The score of logical thinking, which was a KIIS item, was selected as an independent variable $(p<0.05)$. There was a significant difference in the results of analysis of variance $(p<0.05)$, and $R^{2}=$ 0.27 .

\section{Discussion}

\subsection{The Intrinsic Intuitive Ability and Critical Thinking Disposition of Participants}

In contrast to Yamada's study comparing the intuition ability of clinical nurses with disparities in clinical experience years, this study found somewhat different results. There was no significant difference in the total KIIS score between the 
novice and expert nurses, only the KIIS general education score was significantly higher in expert than in novice nurses in this study. However, Yamada et al. mentioned that the KIIS score was significantly higher in nurses with clinical experience of over 10 years than in nurses with clinical experience under 3 years in their study [18]. In Yamada's study, about 3.6 percent of participants had completed nursing college, while half of the novice nurses completed nursing college in this study. In Japan, the Ministry of Education, Culture, Sports, Science and Technology declared the policy of "gakushiryoku," which refers to the competence of university graduates. Part of the competence was special knowledge and creative thinking ability [19]. The educational effect in the nursing college curriculum may influence the KIIS and Critical Thinking Disposition Scale scores of novice nurses, and there were no differences in these scale scores between novice and expert nurses.

\subsection{Comparison of Gaze between Novice and Expert Nurses}

In this study, there were no significant differences in the observation time, total gaze time, and total gaze count of novice and expert nurses. Daikoku's study comparing novice and expert nurses in observation time, gaze time, or gaze count had the same results as this study [8]. In this study, the simulated patient was not operated upon, but was observed in a period immediately after a stroke. Therefore, there was a possibility that the simulated patient suddenly changed. Zeitz claimed that nurses observed patients' state as well as vital signs to find complications immediately after an operation in a study of postoperative patients' observation [20] [21]. It is presumed that both novice and expert nurses observed while assuming the possibility of sudden change. Therefore, it is speculated that the novice nurses were able to observe to a certain level.

In the comparison of proportion of gazing at the gaze items between novice and expert nurses, the proportion of gazing at monitor by novice nurses, and the proportion of gazing at the patient's face, drip route, and bed fence by expert nurses tended to be high. The results of this study agree with those of Suetsugu's study in that nurses or nursing students with little experience tended to pay attention to numerical information [7]. Toanai et al. reported the characteristics of clinical judgment by nurses. In their study, the novice nurses' inferences were limited, so their choice of nursing practices was poor. On the other hand, proficient nurses and expert nurses had more clues and choices for inferring, so they can monitor and confirm patients' state and the correctness of their inferences [11]. It was presumed from the process of novice nurses' clinical judgment that the novice nurses took longer to assess information by collecting information and observing monitor. The proportion of time and count of gazing at the simulated patient's face by expert nurses was higher than for novice nurses. As a result, it was inferred that expert nurses confirmed the patient's changes over time by observing the patient' face. The gaze count of the simulated patient's face was influenced by the participants' logical thinking ability, which was a subcategory 
of the KIIS. Facial expression provides information representing mental states and slight changes in the patient's condition, such as depression after stroke [22]. Because nurses observed simulated patients while considering patient's changes, the nurses' logical thinking related to inferring seemed to influence observation of the ptient's face.

According to a report investigating differences in clinical judgment between novice and expert nurses during patients' care, expert nurses collected more clues to assess patients' problems than novice nurses in the process of nursing care [23]. In this study, the proportion of gazing at the simulated patient's body, such as upper and lower limbs and chest and abdomen by novice nurses tended to be high; in addition, expert nurses tended to gaze at the patient's living environment such as bed fences and drip route. It can be presumed that since the simulated patient had left upper and lower limb paralysis, the expert nurses observed the patient living environment while considering possible dangers such as falling from the bed and pulling out the drip route, beside observation of the simulated patient's disease state.

Both novice and expert nurses hardly gazed at the items of insertion and connection part of bladder indwelling catheter, part of the elastic stocking, part of blood pressure, and pulse oximeter in this study. The cause of not observing these items by novice and expert nurses was that they assess these items to observe later, for example, during care to keep the patient clean, because we told the participants to observe what they need to care for the simulated patient.

It was obvious that the intrinsic intuition ability influenced nurses' eye movements during observation of the simulated patient in this study. General education also affected gaze time and gaze counts during observations of simulated patients. In clinical situations it is necessary to observe and assess within a limited time. It was suggested that contining education to obtain general and special knowledge and critical thinking attitudes is necessary for efficient observation of patients.

\section{Limitation of This Study}

The participants of this study were 19 nurses, so it is considered difficult to generalize the results. It is necessary to increase the participants to raise reliability.

\section{Conclusion}

The results of investigating the nurses' eye movement during observation of patients with disturbed consciousness, showed there was no significant difference in total gaze time and count of novice and expert nurses. The proportion of gazing at monitor by novice nurses tended to be higher, while on the other hand, the proportion of gazing at the simulated patient's face and bed fences by expert nurses tended to be higher. The nurses' critical thinking ability influenced the count of gazing at the simulated patient's face, and this suggests that observing the patient's face may be related to nurses' inferences. 


\section{References}

[1] Tsuchiya, R., Mikami, K., Aizu, K. and Nishizawa, Y. (2013) Patients with Disturbances in Consciousness as Observed by Clinical Nurses: Analysis of Nursing Records. Open Journal of Nursing, 3, 467-471. https://doi.org/10.4236/ojn.2013.37063

[2] Tsuchiya, R., Mikami, K., Aizu, K. and Nishizawa, Y. (2017) Clinical Nurses' Views of Caring for Patients with Consciousness Disturbance: Analysis of Group Interviews with Nurses. Journal of Japan Society of Nursing Research, 40, 613-621. (In Japanese)

[3] Akiba, M., Kumano, H. and Kanno, T. (2008) Visual Attention Point Search System on the Presentation Image. The Institute of Image Information and Television Engineers, 62, 1059-1066. (In Japanese) https://doi.org/10.3169/itej.62.1059

[4] Henneman, A.E., Cunningham, H., Fisher, L.D., Plotkin, K., Nathanson, H.B., Roche, P.J., Marquard, L.J., Reilly, A.C. and Henneman, L.P. (2014) Eye Tracking as a Debriefing Mechanism in the Simulated Setting Improves Patient Safety Practices. Dimensions of Critical Care Nursing, 33, 129-135. https://doi.org/10.1097/DCC.0000000000000041

[5] Browning, M., Cooper, S., Cant, R., Sparkes, L., Bogossian, F., Williams, B., O'Meara, P., Ross, L., Munro, G. and Black, B. (2016) The Use and Limits of Eye-Tracking in High-Fidelity Clinical Scenarios: A Pilot Study. International Emergency Nursing, 25, 43-47. https://doi.org/10.1016/j.ienj.2015.08.002

[6] Koh, Y.I.R., Park, T. Wickens, D.C., Ong, T.L. and Chia, N.S. (2011) Differences in Attentional Strategies by Novice and Experienced Operating Theatre Scrub Nurses. Journal of Experimental Psychology, 17, 233-246. https://doi.org/10.1037/a0025171

[7] Suetsugu, N., Ohki, M. and kaku, T. (2016) Quantitative Analysis of Nursing Observation Employing a Portable Eye-Tracker. Open Journal of Nursing, 6, 53-61. https://doi.org/10.4236/ojn.2016.61006

[8] Daikoku, R. and Saito, Y. (2017) Characteristics of Gaze in Expert Nurses During Observation at the Bedside. Japanese Journal of Nursing Art and Science, 15, 218-226. (In Japanese)

[9] Benner, P. (2001) From Novice to Expert Excellence and Power in Clinical Nursing Practice. Prentice Hall, Englewood Cliff.

[10] Ueno, T., Naito, R., Deguchi, S., Tosa C., Sato, H. and Sato, N. (2002) The Ability of Nurses in Clinical Practice,with a Career of 3 Years and More(The Second Report)-The Ability of Clinical Nursing Practice in Each Age Classes. The Journal of the Japan Academy of Nursing Administration and Policies, 5, 64-70. (In Japanese)

[11] Tonai, M. and Miyakoshi, Y. (2005) Literature Review of Clinical Judgement in Nursing-Elements and Characteristics of Clinical Judgement due to Nurses' Expertise. Japanese Journal of Occupational Medicine and Traumatology, 53, 213-219. (In Japanese)

[12] Fomani, K.F., Cheraghi, A.M., Ghaedi, Y. and Rahimabadi, K.R. (2013) Philosophizing in Skilled Nursing Care: Must or Nice? Open Journal of Nursing, 3, 499-502. https://doi.org/10.4236/ojn.2013.37068

[13] Queiros, J.P.P. (2015) The Knowledge of Expert Nurses and the Practical-Reflective Rationality. Investigación y Educación en Enfermería, 33, 83-91.

[14] Wada, Y., Koyama, S., Honma, S., Matsuoka, H., Kuzuwata, T. and Kuwano, T. (2004) Examination about the Efficiency of Work in the Clinical Nursing. Bulletin of Niigata Seiryo University, 4, 209-218. (In Japanese) 
[15] Yatomi, Y., Inoue, T. and Kawamoto, Y. (2016) Characteristic Duties of Critical Care Nurses in Japan: A Time-Study Comparison with Neurology Ward Nurses. Open Journal of Nursing, 6, 1038-1051. https://doi.org/10.4236/ojn.2016.612099

[16] Kawahara, Y., Sasaki, I., Ogino, M., Kuroda, Y. and Higuchi, Y. (1996) Exploring of the Intrinsic Intuition Ability of Nursing Profession. The Japanese Journal of Health Behavioral Science, 11, 162-177. (In Japanese)

[17] Hirayama, R. and Kusumi, T. (2004) Effect of Critical Thinking Disposition on Interpretation of Controversial Issues: Evaluating Evidences and Drawing Conclusions. The Japanese Journal of Educational Psychology, 52, 186-198. (In Japanese) https://doi.org/10.5926/jjep1953.52.2_186

[18] Yamada, R., Izumi, K., Hiramatsu, T., Kato, M. and Shogenji, M. (2007) Investigation of Clinical Nurses' Intuition Relationships between Hospital, Years of Experience and Nurses' License. The Journal of the Japan Academy of Nursing Administration and Policies, 10, 40-47. (In Japanese)

[19] Central Council for Education (2012) Summary of Report: Towards a Qualitative Transformation of University Education for Building a New Future-Universities Fostering Lifelong Learning and the Ability to Think Independently and Proactively. Ministry of Education, Culture, Sports, Science and Technology.

http://www.mext.go.jp/en/publication/report/title01/detail01/_icsFiles/afieldfile/20 16/12/06/1380275_001.pdf

[20] Zeitz, K. (2003) Nursing Observations during the First 24 Hours after a Surgical Procedure: What Do We So? Journal of Clinical Nursing, 14, 334-343. https://doi.org/10.1111/j.1365-2702.2004.01071.x

[21] Zeitz, K. and McCutcheon, H. (2002) Policies That Drive the Nursing Practice of Postoperative Observations. International Journal of Nursing Studies, 39, 831-839. https://doi.org/10.1016/S0020-7489(02)00024-X

[22] Lee, A.C.K., Tang, S.W., Yu, G.K.K. and Cheung, R.T.F. (2008) The Smiley as a Simple Screening Tool for Depression after Stroke: A Preliminary Study. International Journal of Nursing Studies, 45, 1081-1089. https://doi.org/10.1016/j.ijnurstu.2007.05.008

[23] Hoffman, A.K., Aitken, M.L. and Duffield, C. (2009) A Comparison of Novice and Expert Nurses' Cue Collection during Clinical Decision-Making: Verbal Protocol Analysis. International Journal of Nursing Studies, 46, 1335-1344.

https://doi.org/10.1016/j.ijnurstu.2009.04.001 